Results Of 933 subjects included in the analysis, 59.9\% were symptomatic. Compared to patient infected status, ATV assay clinical sensitivities and specificities were $100 \%$ and $99.0 \%$, respectively, from vaginal swabs; $100 \%$ and $99.4 \%$, respectively, from endocervical swab; $100 \%$ and $99.6 \%$, respectively, from ThinPrep specimens; and $95.2 \%$ and $98.9 \%$, respectively, from patientcollected urine samples. ATV assay performance was similar in asymptomatic and symptomatic patients, by age group (14-17 years and 18 years or older), and was consistent between testing sites. The ATV assay also demonstrated superior performance compared to wet mount microscopic examination and TV culture, regardless of the specimen type utilised.

Conclusions This study provides clinical validation of the ATV assay for the intended uses of detecting TV rRNA in asymptomatic women, and/or to aid in the diagnosis of trichomoniasis in symptomatic women, in a US population. The use of highly accurate, fully-automated molecular tests such as the ATV assay for testing easily obtained vaginal swab and urine samples should facilitate large-scale screening for TV in the US.

\section{P3-S7.09 MUTATIONS ON GYRA OR PARC GENES OF MYCOPLASMA GENITALIUM AND EFFICACIES OF TREATMENT WITH FLUOROQUINOLONES AGAINST M GENITALIUM-RELATED URETHRITIS}

doi:10.1136/sextrans-2011-050108.492

${ }^{1} \mathrm{R}$ Hamasuna, ${ }^{2} \mathrm{~S}$ Takahashi, ${ }^{1} \mathrm{M}$ Matsumoto, ${ }^{1} \mathrm{~T}$ Sho, ${ }^{1} \mathrm{~T}$ Matsumoto. ${ }^{1}$ University of Occupational and Environmental Health, Japan, Kitakyushu, Japan; ${ }^{2}$ Sapporo Medical University of Medicine, Sapporo, Japan

Background Mycoplasma genitalium is one of the pathogens of male urethritis. Macrolides as azithromycin can be the first line-treatment, but macrolides-resistant $M$ genitalium strains were isolated. We tried to use some fluoroquinolones against $M$ genitalium-related urethritis. In these studies, some patients could not treated by fluoroquinolone. In any bacteria, genetic mutations on gyrase genes were related to fluoroquinolone-resistance. In this study, quinoloneresistant determining regions (QRDR) on gyrase genes of $M$ genitalium were analysed and the relationship between the efficacies of fluoroquinolone against $M$ genitalium-related urethritis and genetic mutations on ORDR of $M$ genitalium was examined.

Methods The ORDR on gyrA and parC genes of $M$ genitalium were sequenced and analysed. DNA samples were purified from $M$ genitalium-positive first-voided urine specimens before and after the treatment with fluoroquinolones as gatifloxacin or sitafloxacin. The ORDR of gyrA and parC genes of $M$ genitalium were analysed by using primers according to Shimada's report (Int J Antimicrob Agent, 2010).

Results Twenty-two genomes of $M$ genitalium before the treatment with fluoroquinolones and four genomes from patients with treatment-failure were analysed. Before the treatment, $M$ genitalium genomes have no mutation on gyrA, but had four mutations on parC gene with amino-changes (Ala-69 to Thr, Pro-72 to Ser, Asp-87 to His and Ser-83 to Ile). After the treatment, $M$ genitalium was found in four patients and all remained $M$ genitalium were found mutations on gyrA or parC with amino-changes. $M$ genitalium with mutation on parC (Pro-72 to Ser and Ser-83 to Ile) before treatment was remained. $M$ genitalium with mutation on parC (Pro-72 to Se and Ser-83 to Ile) before treatment was remained and was found additional gyrA mutation (Asp-99 to Asn). In two patients, $M$ genitalium without mutations before treatment remained after treatment. However, these genomes were found with newer mutations on gyrA (Asp-99 to Asn) or on parC (Ser-83 to Ile).

Conclusion From the urine specimens of patients with treatmentfailure of fluoroquinolones, some mutations with amino-change were found on $\mathrm{ORDR}$ of gyrA or $\operatorname{parC}$ genes of $M$ genitalium. It was suggested that these mutations are related with treatment-failure with fluoroquinolones.

\section{P3-S7.10 COMPOSITION OF VAGINAL MICROBIOTA IN BACTERIAL VAGINOSIS PATIENTS AND HEALTHY WOMEN: BASIS FOR GENETIC DIAGNOSIS OF BACTERIAL VAGINOSIS?}

doi:10.1136/sextrans-2011-050108.493

${ }^{1} \mathrm{M}$ Unemo, ${ }^{2} \mathrm{~A}$ Fahlen, ${ }^{3} \mathrm{R}$ Datcu, ${ }^{4} \mathrm{~A}$ Hallen, ${ }^{1} \mathrm{H}$ Fredlund, ${ }^{5} \mathrm{~J}$ Jensen, ${ }^{6} \mathrm{~L}$ Engstrand. ${ }^{1}$ Swedish Reference Laboratory for Pathogenic Neisseria, Örebro, Sweden; ${ }^{2}$ Karolinska Insititute, Sweden; ${ }^{3}$ Statens Serum Institut, Copenhagen, Denmark; ${ }^{4}$ Uppsala University Hospital, Sweden; ${ }^{5}$ Statens Serum Institute, Denmark; ${ }^{6}$ Swedish Institute for Infectious Disease Control, Sweden

Background Bacterial vaginosis (BV) is the most common vaginal infection/disorder. BV is characterised by imbalance in the normal vaginal microbiota with a shift towards higher bacterial diversity and increased $\mathrm{pH}$. The aim of the present study was to describe the differences in vaginal microbiota composition in women suffering from BV compared to healthy women, using massive parallel 454 pyrosequencing.

Methods 163 vaginal samples were collected from women diagnosed with characteristic BV $(n=73)$, women with intermediate BV $(n=11)$, and from healthy women on their regular check-ups $(n=79)$. DNA from the samples was isolated and the bacterial compositions as well as the relative abundance of these bacteria were analysed using 454 pyrosequencing, with GS Titanium amplicons kit (Roche Inc.), of the hypervariable region V4 on the 16S rRNA gene. Finally, 17 different species-specific PCRs were used to verify the species of bacteria found in the 454 pyrosequencing. Results Extensive imbalance of the vaginal microbiota of women with BV compared to healthy controls was revealed. The dominating taxons of the 73 BV cases were Gardnerella, Atopobium, Prevotella, Lactobacillus, Megasphera and Sneathia, while most of the 79 healthy controls had a microbiota totally dominated by Lactobacillus with the BV associated taxons hardly detectable. Furthermore, the 11 patients with intermediate BV predominantly had a mix of the BV associated taxon Gardnerella as well as Lactobacillus. A few of the healthy controls seemed to have a microbiota changing towards the intermediate microflora. Gardnerella may be the first bacteria to establish in the transition from healthy vaginal flora towards a BV associated flora.

Conclusions A clear difference in the composition of the vaginal microbiota between individuals suffering from BV and healthy controls was identified. The present findings are important steps towards the determination of valid potential bacterial markers for $\mathrm{BV}$, are shedding light upon why some women develop BV, as well as show how the microbiota is involved in the development of BV. Knowledge of the composition of the vaginal microbiota is crucial in the development of a BV diagnostic tool and for elucidating appropriate treatment for use in clinical practice.

\section{P3-S7.11 FACTORS ASSOCIATED WITH PERSISTENT BACTERIAL VAGINOSIS AMONG YOUNG REPRODUCTIVE AGE WOMEN IN MYSORE, INDIA}

doi:10.1136/sextrans-2011-050108.494

${ }^{1} \mathrm{P}$ Madhivanan, ${ }^{2} \mathrm{~S}$ Rathod, ${ }^{1} \mathrm{~K}$ Krupp, ${ }^{1} \mathrm{~A}$ Arun, ${ }^{2} \mathrm{~A}$ Reingold, ${ }^{3} \mathrm{C}$ Cohen, ${ }^{3} \mathrm{~J}$ Klausner. ${ }^{1}$ Public Health Research Institute of India, Mysore, India; ${ }^{2}$ University of California, Berkeley, USA; ${ }^{3}$ University of California, San Francisco, USA

Background Bacterial vaginosis (BV) is a common infection and has been associated with adverse health outcomes including preterm birth, pelvic inflammatory disease and acquisition of HIV and other sexually transmitted diseases. There are limited data on persistent 
BV in Indian women. This study examined the risk factors for persistent bacterial vaginosis in young reproductive women in India. Study Design Between November 2005 and January 2007, a prospective cohort study was carried out to examine the relationship of BV and HSV-2 acquisition among women in Mysore. Quarterly, data were collected on sociodemographics, risk behaviour, partner characteristics, followed by a physical examination to diagnose and treat reproductive tract infections. BV was defined using Nugent scoring of gram stained vaginal smears. Persistent BV was calculated using generalised estimating equation methods. Women gave informed consent prior to enrolment in the study.

Results Of the 420 women for which there were data available for all three visits, 114 (27\%) had two or more BV episodes. Women with a history of 2 or more BV episodes were more likely to be infected with Trichomonas vaginalis [OR $72.93,95 \%$ CI 9.69 to 548.4 ] and be diagnosed with HSV-2 infection [OR 2.58, 95\% CI 1.44 to 4.63] compared to women with no BV history. Women with a history of BV were also more likely to report no education, tubal ligation, being a non-Muslim, and having a sex partner who had other sex partners.

Conclusions Young reproductive age women in India have a high persistence of BV. Although the association between BV and Trichomonas vaginalis is unclear, it seems prudent to recommend that women with BV or TV be screened for both infections.

\section{P3-S7.12 INFECTIONS AND INTERVENTIONS IN PREGNANT WOMEN AT HIGH RISK OF PRETERM BIRTH: A COHORT STUDY}

doi:10.1136/sextrans-2011-050108.495

1J Wilson, ${ }^{1}$ I Okpaluba, ${ }^{2} \mathrm{~N}$ Simpson. ' Leeds Teaching Hospitals NHS Trust, Leeds, UK; ${ }^{2}$ University of Leeds, Leeds, UK

Background Preterm birth (PTB) accounts for 65\% neonatal deaths and $50 \%$ neurological disability in children. Prior spontaneous PTB is the highest risk factor for subsequent PTB and is usually associated with sub-clinical infection, possibly due to genetic polymorphisms of proinflammatory cytokines. Bacterial vaginosis (BV) has been implicated in PTB and early treatment may reduce it but less attention has been paid to other infections. We assessed the infective and obstetric complications in a group of pregnant women at high risk of PTB and the interventions to reduce PTB.

Methods Study group: Pregnant women at high risk of PTB Interventions: Microbiological screening for infections from beginning of second trimester then 4-weekly until 28 weeks gestation with treatment of infections found; 2-weekly ultrasound assessment of cervical length during second trimester with cerclage and progesterone injections if needed. Outcome: Gestational age at delivery.

Results We have managed 104 pregnancies in 95 multiparous women who had at least one previous mid-trimester miscarriage (MTM), PTB or stillbirth due to chorioamnionitis. $21 \%$ had two previous MTM and/or PTB; $5 \%$ had three; $4 \%$ had four and $1 \%$ had five MTM/PTB. $75 \%$ were of white ethnicity, $7 \%$ asian, $18 \%$ black. One or more infection was identified in 51 (49\%) of the pregnancies; Group B streptococcal infection (GBS) in $21 \%$, BV in $16 \%$, S aureus in $3 \%$, heavy growth vaginal or urinary coliforms in $16 \%$. Prevalence of infections was more frequent in black (68\%), than white (48\%), than asian (14\%) women. Pregnancy outcome: Exact gestational age unknown in 4 due to transfer to different hospital when $>28 / 40$ gestation so outcomes for 100 pregnancies. Four resulted in MTM leaving 96 viable pregnancies. Gestational age at delivery: 24-27/40 in $3 \% ; 28-31 / 40$ in $3 \% ; 32-37 / 40$ in $13 \%$, term in $81 \%$. There was no association with treated infection and pregnancy outcome, infections had been identified and treated in 39\% of PTBs and $50 \%$ of term births.

Conclusions In this group of women with high risk pregnancies, $49 \%$ had infections known to be associated with PTB which were treated in the second trimester. The overall PTB rate was 19\% with $6 \%$ extremely or very preterm. There was no association between treated infection in pregnancy and PTB. Although the PTB rates are higher than the normal obstetric population they are significantly lower than would be predicted for such high risk pregnancies.

\section{P3-S7.13 EVALUATION OF THE AFFIRM VPIII MICROBIAL IDENTIFICATIONS TEST FOR THE DIAGNOSIS OF VAGINITIS AND BACTERIAL VAGINOSIS}

doi:10.1136/sextrans-2011-050108.496

${ }^{1} \mathrm{D}$ V Maseko, ${ }^{1} \mathrm{~F}$ M Radebe, ${ }^{1} \mathrm{~L}$ Gumede, ${ }^{1} \mathrm{~N}$ Bhojraj Sewphershad, ${ }^{1} \mathrm{~S}$ Khumalo, ${ }^{1}$ I Basson, ${ }^{1} \mathrm{D}$ Lewis. ${ }^{1}$ National Institute for Communicable Diseases (NHLS), Johannesburg, South Africa

Background Vaginitis and bacterial vaginosis (BV) are the most prevalent causes of vaginal symptoms among women of childbearing age. Diagnosis is based on clinical signs and symptoms, microscopy, $\mathrm{pH}$ and the whiff" test, and laboratory performed tests. The Affirm VPIII Microbial Identification test (Becton Dickinson) incorporates non-amplified DNA probes for Candida spp., Trichomonas vaginalis and Gardnerella vaginalis as an indicator for BV.

Objective To compare the Affirm VPIII Microbial Identification test for the detection of Candida spp., Gardnerella vaginalis and Trichomonas vaginalis to graded Gram stain for Bacterial Vaginosis and yeast as well as to an in-house Trichomonas vaginalis PCR (Polymerase Chain Reaction).

Methods In this study, specimens from 191 patients were evaluated. Specimens were collected consecutively from patients with vaginal discharge syndrome presenting at an STI (Sexually transmitted infection) clinic in Gauteng, South Africa between May 2010 and February 2011. Study inclusion was dependent upon the request and collection of both a vaginal swab for Affirm VP III and a second swab to make a smear for Gram staining. A cervical swab specimen was also collected for PCR at the same visit. Graded Gram stain for Bacterial vaginosis and yeast as well as an in-house real-time PCR method on the Rotorgene platform was performed in the laboratory. The sensitivity and specificity of the assay was determined using the graded Gram staining as a gold standard for BV and Candida spp. The real-time PCR was the gold standard for the Trichomonas vaginalis (TV).

Results Analysis of the Affirm VPIII gave a sensitivity of $98 \%$ for BV correctly categorising 80 of the $82 \mathrm{BV}$ positive specimens and specificity of $76 \%$ correctly identifying 38 of the 50 BV- negative specimens. The Affirm VPIII for Candida yielded a sensitivity of $86 \%$ and specificity of $95 \%$. When the Affirm was compared to the Real-time TV PCR the sensitivity was at $45 \%$ and specificity at $99 \%$. Conclusion The Affirm VPIII is an objective system which detects mixed vaginal infections and can be used in any setting. The performance characteristics of the Affirm VPIII for BV and Candida were comparable to those of other published studies. However in this study the Trichomonas vaginalis PCR was used as a gold standard therefore the sensitivity of the Affirm VPII of $45 \%$ is similar to that of culture or wet mount.

\section{P3-S7.14 THE ASSOCIATION OF UREAPLASMA UREALYTICUM WITH MALE NON-GONOCOCCAL URETHRITIS}

doi:10.1136/sextrans-2011-050108.497

${ }^{1} \mathrm{M}$ Frølund, ${ }^{2} \mathrm{P}$ Lidbrink, ${ }^{2} \mathrm{M}$ Cullberg, ${ }^{2} \mathrm{~A}$ Wikström, ${ }^{1} \mathrm{P}$ Ahrens, ${ }^{1} \mathrm{~J} \mathrm{~S}$ Jensen. ${ }^{1}$ Statens Serum Institute, Copenhagen, Denmark; ${ }^{2}$ Karolinska University Hospital, Sweden

Background The aetiology of non-gonococcal urethritis (NGU) is unexplained in $30-50 \%$ of cases. The role of ureaplasmas is not 